\title{
Study of Effects of Blood Amino Acid and Hormone Level for Controlling Triglyceride Accumulation in the Liver of Rats Using Self-Organizing Map
}

\author{
Masato Masuda ${ }^{1}$, Yasushi Nakabayashi ${ }^{1}{ }^{*}$, Ryuji Shioya ${ }^{1}$, Hiroki Nishi ${ }^{2}$, Shinichiro Takahashi ${ }^{2}$, \\ Fumihiko Hakuno ${ }^{2}$ \\ ${ }^{1}$ Center for Computational Mechanics Research, Toyo University, Kawagoe, Japan \\ ${ }^{2}$ Graduate School of Agricultural and Life Sciences, The University of Tokyo, Tokyo, Japan
}

Email address:

nakabayashi@toyo.jp (Y. Nakabayashi)

${ }^{*}$ Corresponding author

\section{To cite this article:}

Masato Masuda, Yasushi Nakabayashi, Ryuji Shioya, Hiroki Nishi, Shinichiro Takahashi, Fumihiko Hakuno. Study of Effects of Blood Amino Acid and Hormone Level for Controlling Triglyceride Accumulation in the Liver of Rats Using Self-Organizing Map. International Journal of Intelligent Information Systems. Vol. 5, No. 6, 2016, pp. 88-93. doi: 10.11648/j.ijiis.20160506.12

Received: August 17,2016; Accepted: November 11, 2016; Published: December 12, 2016

\begin{abstract}
Effects of blood amino acid and hormone level for triglyceride accumulation in the liver are revealed in some previous studies by experimental method. Since there are large individual differences in these effects, it needs a lot of rats for such experiments. Simulation can help, sometimes substitute experiment in various fields especially in engineering field. Recently, combining a supercomputer and Artificial Intelligence technique, simulation is expanding its scope. In this study, one of such a simulation technique, Self-Organizing Map (SOM), proposed by Kohonen and it is a kind of the Neural Networks and using for the competitive learning, is applied for classifying effects of blood amino acid and hormone level for controlling triglyceride accumulation in the liver of rats.
\end{abstract}

Keywords: Amino Acid, Triglyceride, Artificial Intelligence, Self-Organizing Map, Neural Network

\section{Introduction}

Metabolic syndrome is a rapidly growing concern in many countries around the world. It is usually associated with obesity, developing to the risk factors for type-2 diabetes mellitus, atherosclerosis, cardiovascular diseases and hepatic steatosis [1]. A fatty liver is roughly categorized into two types; an alcoholic fatty liver and a non-alcoholic fatty liver. While alcoholic one is caused mainly by continual intake of excess alcohol [2], there known to be many putative triggers for the other one, but the molecular basis is still unclear [3]. Non-alcoholic fatty liver disease (NAFLD), a series of hepatic dysfunctions, is one of the major symptoms of metabolic syndrome and thus there has been a great demand for a better/easier method to treat or diagnose fatty liver.

In the field of nutrition, we and other groups previously reported that dietary protein-malnutrition or protein-restriction caused growth retardation accompanied by hepatic triglyceride (TG) accumulation which was considered to be a kind of NAFLD [4-7]. It is well established that growth retardation is caused at least in part by the reduction of plasma insulin-like growth factor-I (IGF-I) level and actually it was reduced in animals fed a diet with protein-malnutrition or -restriction [4, 8]. However, the mechanisms underlying the hepatic TG accumulation remains to be elucidated. Given that TG accumulation in the liver can be observed only within a few days after the beginning of dietary protein restriction [7] and the liver is the first organ directly detecting the dietary amino acids absorbed in the gut, we hypothesized that the blood amino acid concentration would play important roles in hepatic TG accumulate.

In this study, to evaluate the relationship between a blood amino acid profile and liver TG level, we utilized a machine learning algorithm, Self-Organizing Map (SOM). We fed rats various kinds of experimental diets which have various amino acid-compositions and the data of their blood amino acid concentrations and liver TG was obtained. These animals 
showed a variety of blood amino acid profiles and liver TG levels, not only according to what they ate but also individually and the values had a relatively wide range of variance. Self-Organizing Map, one of the machine learning algorithm first proposed by Kohonen [9], can distinguish and classify such complexed multidimensional data, which we expect can help us understand the relationships between blood amino acid and hepatic TG accumulation. We believe that this approach should give us a novel point of view about fatty liver formation, which should contribute to unveiling the mechanism underlying the progression of NAFLD caused by dietary habits. And also it can lead to practical application to the alternative treatment or prevention of metabolic syndrome.

The rest of the paper is organized as flollows. Section 2 presents experimental data, section 3 describe the detail of SOM. After showing some numerical result, the conclusion is in section 4 .

\section{Experimental Data}

We have already reported that protein malnutrition enhances insulin signaling in rat liver followed by accumulation of hepatic lipid $[6,7]$. In addition, Aoyama et al. have reported that triacylglycerol was accumulated in rat liver fed with low-arginine diet [5]. From these backgrounds, we fed rats with various kinds of nutrition and measured level of blood amino acid, anmonia (NH3), Ornithine (Orn), $\beta$-alanine
(b-Ala), $\alpha$-abscisic acid (a-ABA), Citrulline (Cit), taurine (Tau), Urea and triacylglycerol in liver (Liver TG). 5-week-old male Wistar rats were purchased from Charles River Japan (Kanagawa, Japan). The rats were caged individually and kept in a room maintained at $24 \pm 1{ }^{\circ} \mathrm{C}$ with $50-60 \%$ humidity and a 12h-light (8:00-20:00)/ 12h-dark (20:00-8:00) cycle. They were allowed free access to food and water throughout the experiment. All rats were fed control diet containing $15 \%(\mathrm{w} / \mathrm{w})$ amino acids (detailed composition is shown in Table 1) for 4 days as a training and then divided into some experimental groups. For the next 7 days, each group was given either control diet or experimental diets in which total amino acids or only a specific amino acid was restricted to $1 / 3$ of control diet (Table 1). In the experimental period, body weight and food intake of all rats were measured at 10:00 every day. In collecting the tissues, rats were anesthetized with isoflurane (DS Pharma Animal Health, Japan) and then blood and liver of each rat were obtained. Liver triglyceride was extracted with methanol: chloroform solution $(1: 2, \mathrm{v} / \mathrm{v})$ according to the Folch's method [11] and quantified using Triglycerid E-test Wako (WAKO, Japan). Blood amino acid concentration was measured using Amino Acid Analyzer L-8900 (Hitachi Hi-Tech, Japan) according to the manufacturer's protocol. All animal care and experiments conformed to the Guidelines for Animal Experiments of The University of Tokyo and were approved by the Animal Research Committee of The University of Tokyo.

Table 1. The composition of diets for rats: The composition of diet $(\mathrm{g} / \mathrm{kg})$ for each rat was shown. Soybean oil (50 $\mathrm{g} / \mathrm{kg})$, vitamin mixture (10 $\mathrm{g} / \mathrm{kg})$, mineral mixture $(40 \mathrm{~g} / \mathrm{kg})$ and cellulose powder $(100 \mathrm{~g} / \mathrm{kg})$ were added to each diet.

\begin{tabular}{|c|c|c|c|c|c|c|c|c|c|c|c|c|}
\hline & Rat No. & Ile & Leu & Lys & Met & Cys & Phe & Tyr & Thr & Trp & Val & His \\
\hline $\mathrm{CN}$ & $1,2,3,28,29,30,40,41,42,43,44$ & 7.1 & 13.0 & 14.1 & 6.4 & 0.8 & 7.2 & 7.8 & 6.1 & 1.7 & 9.2 & 4.1 \\
\hline$\Delta$ Arg & $4,5,6,55,56,57,58,59,60,61,62,63$ & 7.1 & 13.0 & 14.1 & 6.4 & 0.8 & 7.2 & 7.8 & 6.1 & 1.7 & 9.2 & 4.1 \\
\hline$\Delta \mathrm{R} / \mathrm{SA}$ & $7,8,9$ & 7.1 & 13.0 & 14.1 & 2.1 & 0.3 & 7.2 & 7.8 & 6.1 & 1.7 & 9.2 & 4.1 \\
\hline$\triangle \mathrm{R} / \mathrm{BCA}$ & $10,11,12$ & 2.4 & 4.3 & 14.1 & 6.4 & 0.8 & 7.2 & 7.8 & 6.1 & 1.7 & 3.1 & 4.1 \\
\hline$\Delta \mathrm{R} / \mathrm{AA}$ & $13,14,15$ & 7.1 & 13.0 & 14.1 & 6.4 & 0.8 & 7.2 & 7.8 & 6.1 & 1.7 & 9.2 & 4.1 \\
\hline$\Delta \mathrm{R} / \mathrm{BA}$ & $16,17,18$ & 7.1 & 13.0 & 4.7 & 6.4 & 0.8 & 7.2 & 7.8 & 6.1 & 1.7 & 9.2 & 1.4 \\
\hline$\Delta \mathrm{R} / \mathrm{NA}$ & $19,20,21$ & 7.1 & 13.0 & 14.1 & 6.4 & 0.8 & 2.4 & 2.6 & 2.0 & 0.6 & 9.2 & 4.1 \\
\hline $5 \mathrm{AA}+\mathrm{T}$ & $25,26,27$ & 2.4 & 4.3 & 4.7 & 2.1 & 0.3 & 2.4 & 2.6 & 6.1 & 0.6 & 3.1 & 1.4 \\
\hline$\Delta \mathrm{Tyr}$ & $31,32,33$ & 7.1 & 13.0 & 14.1 & 6.4 & 0.8 & 7.2 & 2.6 & 6.1 & 1.7 & 9.2 & 4.1 \\
\hline H-Met & $34,35,36$ & 7.1 & 13.0 & 14.1 & 30.0 & 0.8 & 7.2 & 7.8 & 6.1 & 1.7 & 9.2 & 4.1 \\
\hline $\mathrm{H}-\mathrm{His}$ & $37,38,39$ & 7.1 & 13.0 & 14.1 & 6.4 & 0.8 & 7.2 & 7.8 & 6.1 & 1.7 & 9.2 & 30.0 \\
\hline$\Delta$ Lys & $50,51,52,53,54$ & 7.1 & 13.0 & 4.7 & 6.4 & 0.8 & 7.2 & 7.8 & 6.1 & 1.7 & 9.2 & 4.1 \\
\hline
\end{tabular}

Table 1. Continue.

\begin{tabular}{|c|c|c|c|c|c|c|c|c|c|c|c|}
\hline & Rat No. & Arg & Ala & Asp & Asn & Glu & Gly & Pro & Ser & Gln & corn starch \\
\hline $\mathrm{CN}$ & $1,2,3,28,29,30,40,41,42,43,44$ & 5.2 & 4.1 & 5.1 & 5.8 & 14.6 & 2.6 & 15.0 & 8.1 & 14.6 & 647.5 \\
\hline$\Delta \mathrm{Arg}$ & $4,5,6,55,56,57,58,59,60,61,62,63$ & 1.7 & 4.1 & 5.1 & 5.8 & 14.6 & 2.6 & 15.0 & 8.1 & 14.6 & 651.0 \\
\hline$\Delta \mathrm{R} / \mathrm{SA}$ & $7,8,9$ & 1.7 & 4.1 & 5.1 & 5.8 & 14.6 & 2.6 & 15.0 & 8.1 & 14.6 & 655.7 \\
\hline$\Delta \mathrm{R} / \mathrm{BCA}$ & $10,11,12$ & 1.7 & 4.1 & 5.1 & 5.8 & 14.6 & 2.6 & 15.0 & 8.1 & 14.6 & 670.4 \\
\hline$\Delta \mathrm{R} / \mathrm{AA}$ & $13,14,15$ & 1.7 & 4.1 & 1.7 & 5.8 & 4.9 & 2.6 & 15.0 & 8.1 & 14.6 & 644.0 \\
\hline$\Delta \mathrm{R} / \mathrm{BA}$ & $16,17,18$ & 1.7 & 4.1 & 5.1 & 5.8 & 14.6 & 2.6 & 15.0 & 8.1 & 14.6 & 633.0 \\
\hline$\Delta \mathrm{R} / \mathrm{NA}$ & $19,20,21$ & 1.7 & 1.4 & 5.1 & 1.9 & 14.6 & 0.9 & 5.0 & 2.7 & 4.9 & 699.5 \\
\hline $5 \mathrm{AA}+\mathrm{T}$ & $25,26,27$ & 1.7 & 1.4 & 5.1 & 1.9 & 4.9 & 0.9 & 5.0 & 2.7 & 4.9 & 745.1 \\
\hline$\Delta \mathrm{Tyr}$ & $31,32,33$ & 5.2 & 4.1 & 5.1 & 5.8 & 14.6 & 2.6 & 15.0 & 8.1 & 14.6 & 647.5 \\
\hline H-Met & $34,35,36$ & 5.2 & 4.1 & 5.1 & 5.8 & 14.6 & 2.6 & 15.0 & 8.1 & 14.6 & 647.5 \\
\hline $\mathrm{H}-\mathrm{His}$ & $37,38,39$ & 5.2 & 4.1 & 5.1 & 5.8 & 14.6 & 2.6 & 15.0 & 8.1 & 14.6 & 647.5 \\
\hline$\Delta$ Lys & $50,51,52,53,54$ & 5.2 & 4.1 & 5.1 & 5.8 & 14.6 & 2.6 & 15.0 & 8.1 & 14.6 & 656.9 \\
\hline
\end{tabular}




\section{SOM}

\subsection{Outline of SOM}

SOM, which is one of neural networks and was proposed by T. Kohonen [9], is a two-layered unsupervised competitive learning model that does not have a hidden layer. In the learning algorithm of SOM, the characteristics of input data are learned through neighborhood learning. A map is formed so that similar kinds of data are located in the neighborhood and other data are located at distant places. Accordingly, it is possible to visualize high-dimensional vector data, and understand the relations among data intuitively.

As an example [9], the animals were classified. These characteristics of Table 2 are classified on the map (Fig. 1).

Table 2. Input Vectors of Animals.

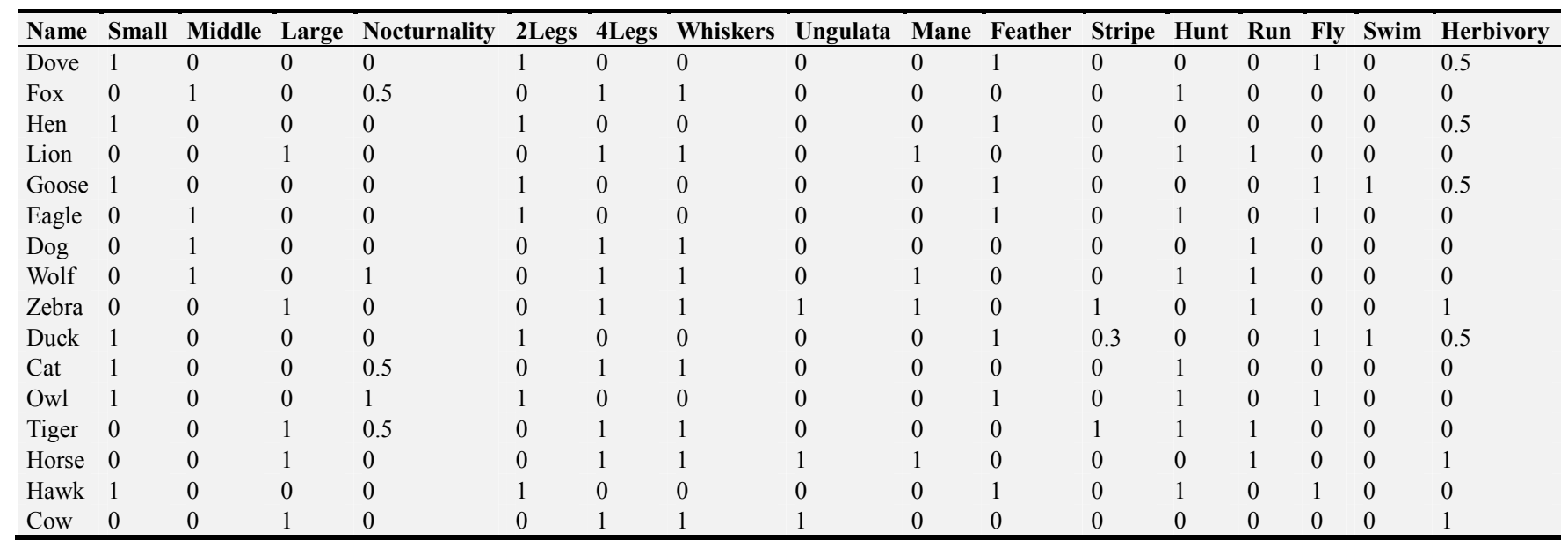

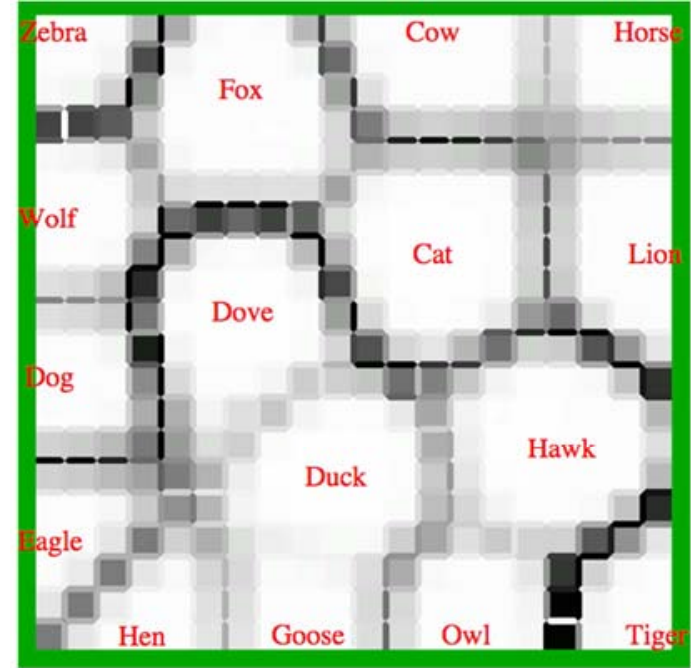

Figure 1. Classification of Animals (16 kinds of animals with 16-dimensional characteristics).

\subsection{SOM Algorithm}

SOM has a map with the $\mathrm{M} \times \mathrm{N}$ units ( $\mathrm{M}$ is the number of unit in horizontal direction, $\mathrm{N}$ is the number of unit in vertical derection), and weighted units exist (Fig. 2). Unit weights are updated in neighborhood learning. The algorithm of SOM [9, 10] is as follows:

Step 1: Initialize the weight vector w of a unit.

Step 2: Input the vector

$\mathrm{x}_{\text {class }}=\left(x_{\text {class }}^{1}, x_{\text {class }}^{2}, x_{\text {class }}^{3}, \cdots, x_{\text {class }}^{I}\right)$ into input layer. Here, "class" represents the number of input vectors, and "I" denotes the dimension of the input vector.
Step 3: Calculate the Euclidean distance "Dist" between an input vector and the weight vector of a unit in the map layer (competitive layer).

$$
\text { Dist }_{m}=\sqrt{\sum_{i}\left(x_{\text {class }}^{i}-w_{m}^{i}\right)^{2}}
$$

Here, "m" represents the number of units from 1 to $M \times N$, "i" represents the number of dimensions of vector $\mathrm{x}_{\text {class }}$.

Step 4: Define the unit that minimizes the Euclidean distance obtained at Step 3 as the winning unit BMU (Best Matching Unit).

$$
B M U=\arg \min _{m} D_{i s t_{m}}
$$

Step 5: Update the weights of the winning unit and surrounding units only.

$$
\begin{gathered}
\Delta w=h(l(B M U, m))\left(x_{\text {class }}^{i}-w_{m}^{i}\right) \\
h(l)=\alpha \exp \left(-\frac{l^{2}}{2 \sigma^{2}}\right)
\end{gathered}
$$

Step 6: Repeat Steps 2 to 5 sufficiently.

Here, 1 represents the distance between the winning unit and the unit whose weight is updated. $h$ and $\sigma$ denote the neighborhood function and the range of the influence of the neighborhood function (neighborhood radius), respectively. This is decreased according to learning steps. $\alpha$ is learning coefficient, ranging from 0 to 1 . The learning coefficient $\alpha$ decreases as learning steps proceed. 


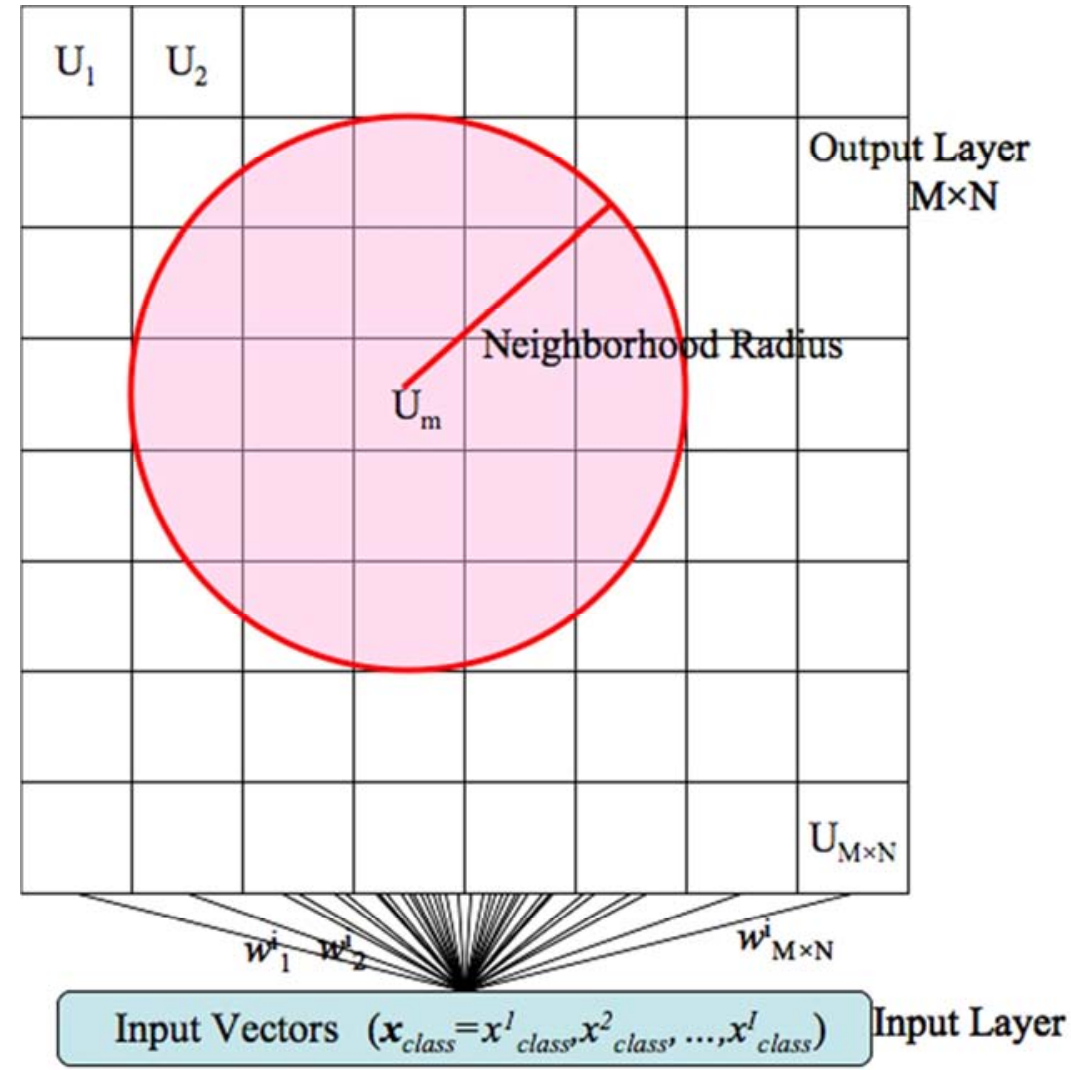

Figure 2. Conceptual Diagram of SOM (U is map unit, $m$ is unit number, $w$ is uniting weight, X is input signal)).

\subsection{Input Data}

Input Data given to SOM is created from the experimental data. Used data are blood amino acid in the rats and quantity of Triglyceride in the liver (Liver TG). Amino acid has 22 kinds, and the input data has 23 characteristics together with Triglyceride in the liver. Input of SOM is 23 dimensions vector combined the blood amino acids and the Liver TG of each rat. Table 2 shows a part of input data. This can make it possible to estimate Liver TG by quantity of blood amino acid in a rat. Fig. 3 shows the average values, the maximum values, and minimum values of the data.

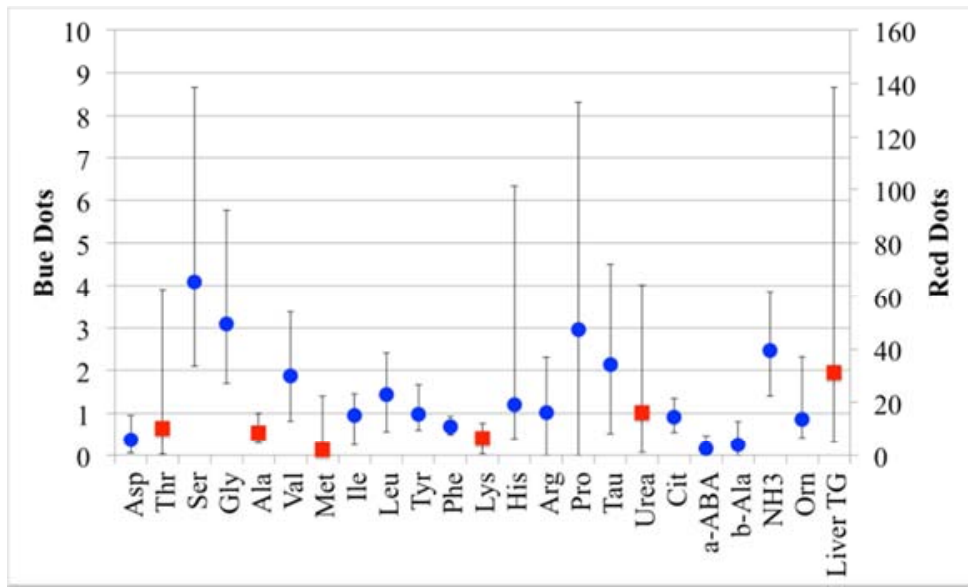

Figure 3. Graph of Amino Acid and Liver TG (Dots are average values of each factor. Error bars respectively the maximum and minimum values).

\subsection{Learning $S O M$}

In the SOM learning, map size was $30 \times 30$, and it was changed the neighborhood radius using RPSOM (Radius Parallel SOM) [12]. This method is similar to the temperature parallel Simulated Annealing Method. Several neighborhood radii are calculated in parallel, and the better neighborhood radius case is selected so as to satisfy the each evaluation value of the Fitness and the Interpolation. In this case, neighborhood radii were used three radii from 2.0 to 1.0 , from 5.0 to 2.0 and from 8.0 to 3.0 . 


\subsection{Result}

Fig. 4 show the learning result. The height direction of the figure represents the value of the Liver TG. At the center bottom of the map, there is mass of rats from No.22 to 27 . At the center of left, cluster of no.40 45 and 50 55 is formed. These are judged these have similar characteristics. Table 3 indicates the quantity of blood amino acid and Liver TG of No.22, 25, and 27 and No.40, 43, and 50. They are standardized at 0 to 1 . Compared with No. 22, 25, and 27, Ser and Ala are respectively different. In addition, there are several items different from other two in each individual. It can be concluded they are very similar vectors on the whole. No.40, 43, and 50 were compared. Similarly, these also have the slightly different item respectively. There is individual difference, but it is a vector that is roughly similar. And Liver $\mathrm{TG}$ is also generally similar. From the FIg. 4(b), the classification map of Liver TG that has transferred smoothly was made.

Table 3. Blood Amino acid and Liver TG of rats.

\begin{tabular}{llllllll}
\hline \multirow{2}{*}{ Rat Number } & \multicolumn{2}{l}{ Blood Amino Acid } & & & & \\
\cline { 2 - 6 } & Asp & Thr & Ser & $\ldots$ & b-Ala & NH3 & Orn \\
\hline No.1 & 0.119 & 5.693 & 2.382 & $\ldots$ & 0.071 & 2.135 & 0.43 \\
No.2 & 0.122 & 7.698 & 3.342 & $\ldots$ & 0.059 & 1.954 & 0.71 \\
No.3 & 0.143 & 6.058 & 3.081 & $\ldots$ & 0.071 & 1.676 & 0.469 \\
No.4 & 0.113 & 5.014 & 3.044 & $\ldots$ & 0.05 & 2.379 & 0.53 \\
No.60 & 0.098 & 6.48 & 2.444 & $\ldots$ & 0.064 & 2.623 & 0.513 \\
No.61 & 0.075 & 3.815 & 2.971 & $\ldots$ & 0.049 & 2.558 & 0.486 \\
No.62 & 0.088 & 6.248 & 3.266 & $\ldots$ & 0.059 & 2.089 & $5.339 \times 10^{1}$ \\
No.63 & 0.053 & 5.424 & 2.098 & $\ldots$ & 0.058 & 2.455 & 0.48 \\
\hline
\end{tabular}

Table 4. Blood Amino acid and Liver TG of No.22, 25, 27 and No.41, 43, 55.

\begin{tabular}{|c|c|c|c|c|c|c|c|c|c|c|c|c|}
\hline & Asp & Thr & Ser & Gly & Ala & Val & Met & Ile & Leu & Tyr & Phe & Lys \\
\hline No. 22 & 0.104 & 0.924 & 0.896 & 0.852 & 0.714 & 0.482 & 0.044 & 0.407 & 0.420 & 0.404 & 0.651 & 0.317 \\
\hline No. 25 & 0.115 & 1.000 & 1.000 & 1.000 & 0.844 & 0.525 & 0.041 & 0.547 & 0.535 & 0.443 & 0.725 & 0.387 \\
\hline No. 27 & 0.103 & 0.916 & 0.743 & 0.823 & 0.590 & 0.469 & 0.022 & 0.501 & 0.450 & 0.370 & 0.731 & 0.280 \\
\hline No. 22 & 0.160 & 0.254 & 0.503 & 0.154 & 0.018 & 0.693 & 0.692 & 0.084 & 0.518 & 0.183 & 0.177 & \\
\hline No. 25 & 0.159 & 0.232 & 0.525 & 0.190 & 0.025 & 0.653 & 0.672 & 0.088 & 0.497 & 0.208 & 0.253 & \\
\hline No. 27 & 0.123 & 0.294 & 0.283 & 0.152 & 0.023 & 0.655 & 0.605 & 0.076 & 0.364 & 0.194 & 0.231 & \\
\hline No.40 & 0.950 & 0.086 & 0.378 & 0.671 & 0.469 & 0.476 & 0.035 & 0.744 & 0.567 & 0.711 & 0.782 & 0.456 \\
\hline No.43 & 0.835 & 0.127 & 0.395 & 0.543 & 0.478 & 0.506 & 0.029 & 0.793 & 0.603 & 0.654 & 0.857 & 0.524 \\
\hline \multirow[t]{2}{*}{ No.50 } & 0.915 & 0.156 & 0.542 & 0.609 & 0.537 & 0.668 & 0.045 & 0.880 & 0.649 & 0.591 & 0.812 & 0.111 \\
\hline & His & Arg & Pro & Tau & Urea & Cit & $\mathrm{a}-\mathrm{ABA}$ & b-Ala & NH3 & Orn & Liver TG & \\
\hline No.40 & 0.118 & 0.773 & 0.000 & 0.877 & 0.151 & 0.607 & 0.343 & 0.646 & 0.776 & 0.169 & 0.083 & \\
\hline No.43 & 0.122 & 0.896 & 0.000 & 0.818 & 0.113 & 0.698 & 0.338 & 0.766 & 0.629 & 0.582 & 0.153 & \\
\hline No. 50 & 0.108 & 0.718 & 0.451 & 0.904 & 0.483 & 0.648 & 0.529 & 1.000 & 0.663 & 0.562 & 0.077 & \\
\hline
\end{tabular}

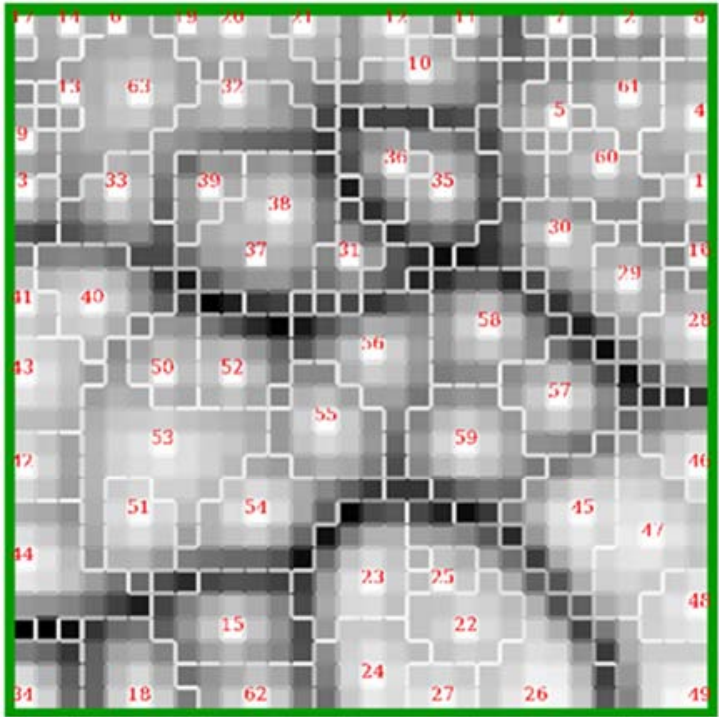

(a) All input relationship

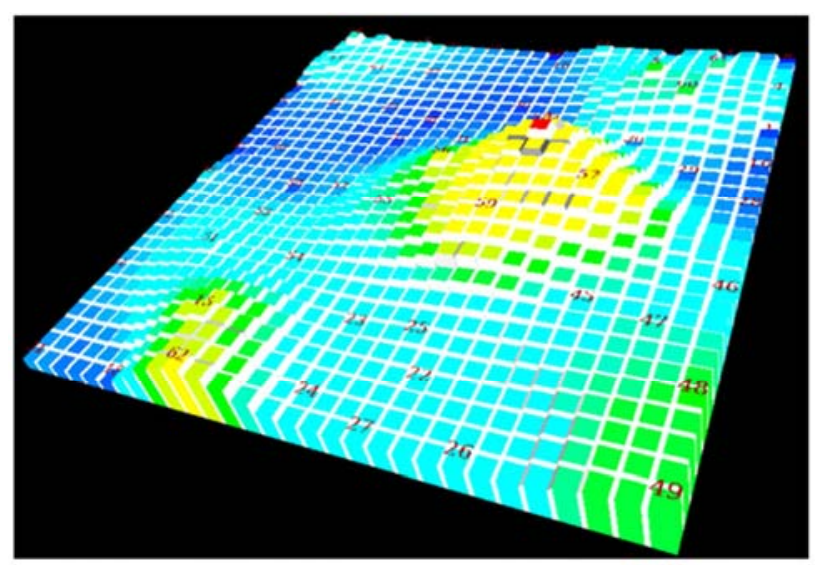

(b) Color Map of Liver TG

Figure 4. Result of RPSOM (Map Size is 30×30, Input Vectors are 23, Learning Iterations are 1,000 times. (a) Color thickness indicates the distance from an input vector. The thin line denotes the distance between adjacent units. As it is nearer, the color whitens, and vice versa. (b)Height is Liver TG). 


\section{Conclusion}

We tried to classify and estimate relation between the quantity of blood amino acid and Liver TG using the proposed method "Radius Parallel SOM". As the results, we obtained the following remarks:

(1) The Self-Organizing Map, which use the blood amino acid and Liver TG as input data and is classified by the RPSOM, was formed.

(2) As rat No.22 to 27 and No.40 to 45,50 to 55 , when quantity of blood amino acid are similar, Liver TG are also similar.

(3) The classification map of Liver TG that has transferred smoothly was made.

(4) It was possible to visually understand the relationship of quantity of blood amino acid and Liver TG using RPSOM. And that was classified into a similar group.

(5) In the future, it will be necessary to consider predicting Liver TG from unknown input data and prediction method.

\section{References}

[1] R. Bass and I. Eneli, "Severe childhood obesity: an under-recognised and growing health problem," Postgr. Med J, vol. 91, no. 1081, pp. 639-645, 2015.

[2] F. A. Lívero and A. Acco, "Molecular basis of alcoholic fatty liver disease: From incidence to treatment," Hepatol. Res., 2015.

[3] M. Hernandez-Rodas, R. Valenzuela, and L. Videla, "Relevant Aspects of Nutritional and Dietary Interventions in Non-Alcoholic Fatty Liver Disease,” Int. J. Mol. Sci., vol. 16, no. 10, pp. 25168-25198, 2015.

[4] A. Takenaka, M. Mori, S. Yamada, J. Ohgane, S. Takahashi, and T. Noguchi, "Nutritional regulation of gene expression of insulin-like growth factor-binding proteins and the acid-labile subunit in various tissues of rats," J Endocrinol, vol. 150, no. 1, pp. 33-41, 1996.

[5] Y. Aoyama, A. Yoshida, and K. Ashida, "Effect of Some Dietary Additions to Either an Arginine- Devoid Diet or a Diet Supplemented with Orotic Acid Refed after Starvation on Liver Lipid Content during Essential Fatty Acid Deficiency in Rats," J. Nutr., vol. 111, pp. 895-906, 1981.

[6] Y. Toyoshima, R. Tokita, Y. Taguchi, N. Akiyama-akanishi, and A. Takenaka, "Tissue-specific effects of protein malnutrition on insulin signaling pathway and lipid accumulation in growing rats," Endocr. J., vol. 61, no. 5, pp. 499-512, 2014.

[7] Y. Ozaki, T. Takeda, N. Akanishi, F. Hakuno, Y. Toyoshima, S.-I Takahashi, and A. Takenaka, "Insulin injection restored increased insulin receptor substrate (IRS)-2 protein during short-term protein restriction but did not affect reduced insulin-like growth factor (IGF)-I mRNA or increased triglyceride accumulation in the liver of rats.," Biosci. Biotechnol. Biochem., vol. 78, no. 1, pp. 130-138, 2014.

[8] A. Takenaka, N. Oki, S. I. Takahashi, and T. Noguchi, "Dietary restriction of single essential amino acids reduces plasma insulin-like growth factor-I (IGF-I) but does not affect plasma IGF-binding protein-1 in rats.," J. Nutr., vol. 130, no. September, pp. 2910-2914, 2000.

[9] T. Kohonen, "Self-Organized formation of topologically correct feature maps”, Biological Cybernetics, Vol.43, pp.59-69, 1982.

[10] T. Kohonen, "Self-Organizing Maps", Springer-Verlag, 1995.

[11] Folch J, Lees M, Sloane Stanley GH, A simple method for the isolation and purification of total lipids from animal tissues. J. Biol. Chem. 226: 497-509, (1957).

[12] Masato MASUDA, Yasushi NAKABAYASHI and Genki YAGAWA, Radius Parallel Self- Organizing Map (RPSOM), Journal of Computational Science and Technology, Vol. 6, No. 1, 16-27, DOI: 10.1299/jcst.6.16 (2012). 